PRACTICE CMAJ

\title{
Eschar: a clue to scrub typhus
}

\author{
Chih-Chung Shiao MD, Seng-Yi Lin MD
}

Competing interests: None declared.

This article has been peer reviewed.

Affiliations: From the Divisions of Nephrology (Shiao) and Infection (Lin), Department of Internal Medicine, Saint Mary's Hospital, Luodong, Taiwan; and Saint Mary's Medicine, Nursing and Management College (Shiao), Yilan, Taiwan

Correspondence to: Dr. Chih-Chung Shiao, chungyy2001@yahoo .com.tw

CMAJ 2011. DOI:10.1503 /cmaj.101929

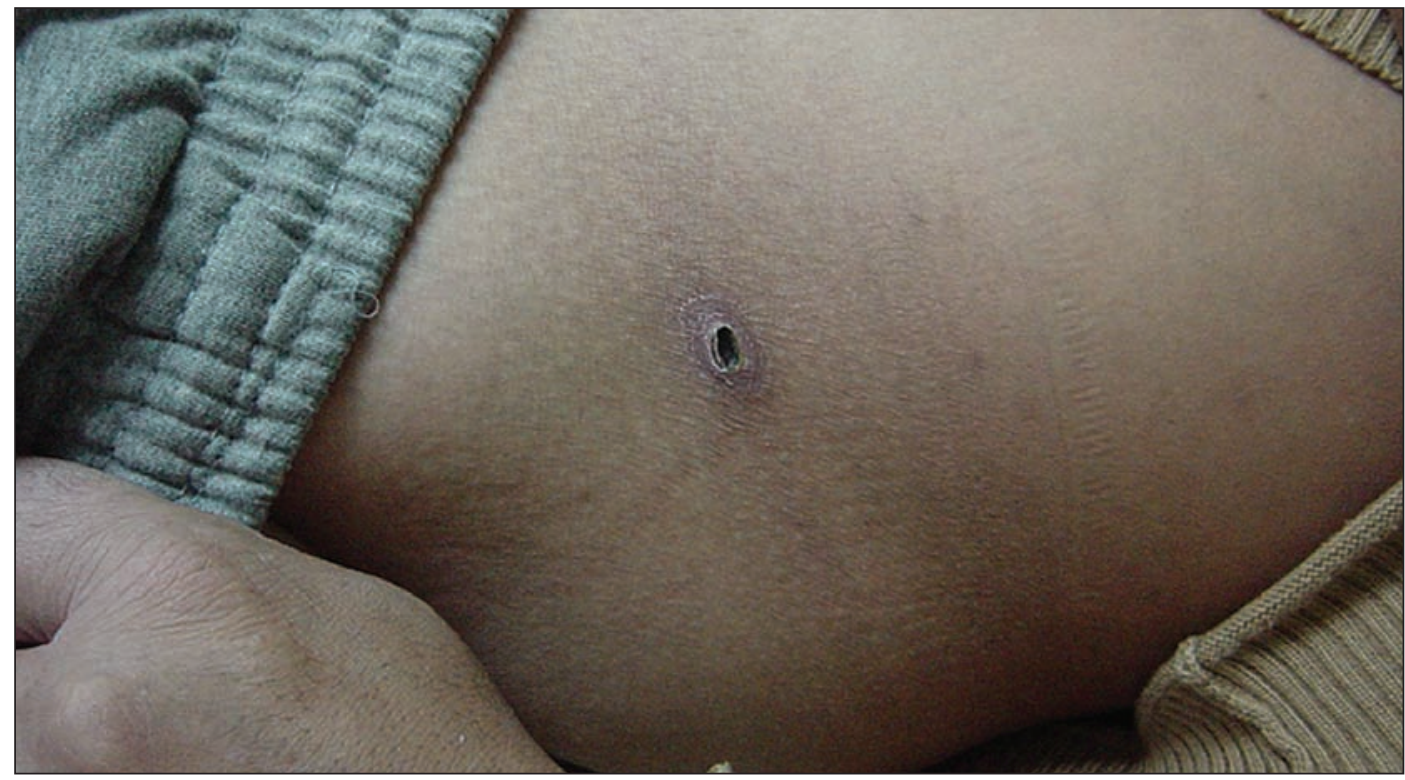

Figure 1: Lower abdomen of a 33-year-old febrile man from a rural Taiwanese village showing an eschar, a necrotic lesion induced by a mite bite, measuring $15 \mathrm{~mm}$.

A 33-year-old man from a rural Taiwanese village was admitted with a three-day history of fever, headache, muscle pain and skin rashes. On examination, he was febrile $\left(39.2^{\circ} \mathrm{C}\right)$ with generalized macular patches and an eschar measuring $15 \mathrm{~mm}$ on the left lower quadrant of his abdomen (Figure 1). His leukocyte count was normal, but he had an elevated C-reactive protein level of 83 (normal 0-8) $\mathrm{mg} / \mathrm{L}$, an aspartate aminotransferase level of 183 (normal 0-37) U/L and an alanine aminotransferase level of 166 (normal 041) U/L. His blood cultures showed no growth.

Based on a working diagnosis of scrub typhus, we prescribed doxycycline. The patient's condition improved dramatically within two days, and he had a full clinical recovery by the eighth day of treatment. The diagnosis of scrub typhus was confirmed serologically using the indirect fluorescent method, with a greater than fourfold increase in antibody titers between two samples drawn two weeks apart. ${ }^{1}$

Scrub typhus is a potentially fatal mite-borne rickettsial infection caused by Orientia tsutsugamushi. It is endemic to the Asia-Pacific region, which has an estimated 1 million instances per year. ${ }^{2}$ Among North American travellers returning from Asia, rickettsial diseases account for $1.0 \%-$ $1.6 \%$ of febrile illness. ${ }^{3}$ Those affected may have headache, myalgias, hearing loss and rash, in addition to fever. Encephalitis, hepatitis, and pulmonary and cardiac involvement can occur. ${ }^{2,4}$

Scrub typhus is often diagnosed clinically based on exposure to endemic regions and its characteristic eschar, which usually appears on the lower extremities, axillae or genital region., ${ }^{2,4}$ Still, the diagnosis can be tricky, and similar eschars can be caused by spider bites, Mediterranean spotted fever, Queensland tick typhus, African tick-bite fever and anthrax (Appendix 1, available at www.cmaj.ca /lookup/suppl/doi:10.1503/cmaj.101929/-/DC1). Early recognition and prompt treatment with empirical doxycycline can be life-saving.

\section{References}

1. Koh GC, Maude RJ, Paris DH, et al. Diagnosis of scrub typhus. Am J Trop Med Hyg 2010;82:368-70.

2. Botelho-Nevers E, Raoult D. Fever of unknown origin due to rickettsioses. Infect Dis Clin North Am 2007;21:997-1011.

3. Freedman DO, Weld LH, Kozarsky PE, et al. Spectrum of disease and relation to place of exposure among ill returned travelers. N Engl J Med 2006;354:119-30.

4. Hendershot EF, Sexton DJ. Scrub typhus and rickettsial diseases in international travelers: a review. Curr Infect Dis Rep 2009;11:66-72. 